\title{
Nonclassical Approximate Symmetries of Evolution Equations with a Small Parameter
}

\author{
Svetlana KORDYUKOVA
}

Department of Mathematics, Ufa State Aviation Technical University,

12 K. Marx Str., Ufa, 450000 Russia

E-mail:sveta.kor05@mail.ru

Received November 30, 2005, in final form March 17, 2006; Published online April 10, 2006

Original article is available at http://www.emis.de/journals/SIGMA/2006/Paper040/

\begin{abstract}
We introduce a method of approximate nonclassical Lie-Bäcklund symmetries for partial differential equations with a small parameter and discuss applications of this method to finding of approximate solutions both integrable and nonintegrable equations.
\end{abstract}

Key words: nonclassical Lie-Bäcklund symmetries; approximate symmetry; conditionalinvariant solution

2000 Mathematics Subject Classification: 58J70; 35Q53

\section{Introduction}

The theory of one- and multi-parameter approximate transformation groups was initiated by Ibragimov, Baikov, Gazizov [1, 13]. They introduced the notion of approximate Lie-Bäcklund symmetry of a partial differential equation with a small parameter $\varepsilon$ and develop a method, which allows to construct approximate Lie-Bäcklund symmetries of such an equation (a perturbed equation) in the form of a power series in $\varepsilon$, starting from an exact Lie-Bäcklund symmetry of the unperturbed equation (for $\varepsilon=0$ ). Similar ideas were suggested independently by Fushchych and Shtelen (see, for instance, [5] and the bibliography therein). The main purpose of this paper is to extend these methods to approximate nonclassical Lie-Bäcklund symmetries.

Nonclassical symmetries appeared for the first time in the paper by Bluman and Cole in 1969 [2]. Since then this theory was actively developed in papers of: Olver and Rosenau [3] (nonclassical method), Clarkson and Kruskal [4] (nonclassical symmetry reductions (direct method)), Fushchych's school ([5] and the bibliography therein) (conditional symmetries and reductions of partial differential equations), Fokas and Liu [6] (the generalized conditional symmetry method), Olver [10] (nonclassical and conditional symmetries). Nonclassical Lie-Bäcklund symmetries for evolution equations were considered in the paper by Zhdanov [7]. This paper also contains a theorem on reduction of an evolution equation to a system of ordinary differential equations. The notion of nonclassical Lie-Bäcklund symmetry is a very wide generalization of the notion of point symmetry. Nevertheless, in many cases, nonclassical Lie-Bäcklund symmetries enable to construct differential substitutions, which reduce a partial differential equation to a system of ordinary differential equations. This fact is used for finding new solutions of partial differential equations, which cannot be found with the help of the classical symmetry method.

The method of approximate conditional symmetries for partial differential equations with a small parameter was suggested by Mahomed and Qu [8] (point symmetries), Kara, Mahomed and $\mathrm{Qu}$ (potential approximate symmetries) [9]. In this paper we develop the method of approximate nonclassical Lie-Bäcklund symmetries. In [1], Baikov, Gazizov and Ibragimov constructed approximate Lie-Bäcklund symmetries of the Korteweg-de Vries equation $u_{t}=u u_{x}+\varepsilon u_{x x x}$, 
starting from exact symmetries of the transport equation $u_{t}=u u_{x}$. In this paper, we extend this construction to approximate nonclassical Lie-Bäcklund symmetries.

We will consider a particular class of evolution partial differential equations with a small parameter given by

$$
u_{t}=u u_{x}+\varepsilon H\left(t, x, u, u_{x}, u_{x x}, \ldots\right) .
$$

This class contains both integrable and nonintegrable equations. We consider such equations as perturbations of the transport equation $u_{t}=u u_{x}$ and construct approximate nonclassical symmetries of these equations, starting from exact nonclassical symmetries of the transport equation. Using these approximate nonclassical symmetries and the reduction theorem, we find approximate conditionally invariant solutions of equations under consideration. As an example, we find approximate solutions of the $\mathrm{KdV}$ equation with a small parameter and of some nonintegrable equations.

\section{Nonclassical Lie-Bäcklund symmetries}

Recall the definition of classical Lie-Bäcklund symmetries (here we will consider symmetries given by canonical Lie-Bäcklund operators):

Definition 1. An operator

$$
X=\zeta \frac{\partial}{\partial u}+\left(D_{x} \zeta\right) \frac{\partial}{\partial u_{x}}+\left(D_{t} \zeta\right) \frac{\partial}{\partial u_{t}}+\left(D_{x x} \zeta\right) \frac{\partial}{\partial u_{x x}}+\cdots
$$

where

$$
\zeta=\zeta\left(t, x, u, u_{x}, u_{x x}, \ldots\right),
$$

will be called a classical Lie-Bäcklund symmetry for a partial differential equation of evolution type

$$
u_{t}=F\left(t, x, u, u_{x}, u_{x x}, \ldots\right),
$$

if

$$
\left.X\left(u_{t}-F\right)\right|_{u_{t}=F}=0 .
$$

Here $D_{x}$ and $D_{t}$ are the total differentiation operators:

$$
\begin{aligned}
& D_{x}=\partial_{x}+\partial_{u} u_{x}+\partial_{u_{x}} u_{x x}+\partial_{u_{x x}} u_{x x x}+\cdots, \\
& D_{t}=\partial_{t}+\partial_{u} u_{t}+\partial_{u_{x}} u_{x t}+\partial_{u_{x x}} u_{x x t}+\cdots,
\end{aligned}
$$

$D_{x x}=D_{x}^{2}=D_{x}\left(D_{x}\right), D_{x x x}=D_{x}^{3}=D_{x}\left(D_{x x}\right)$ etc. The equation (1) is the determining equation for Lie-Bäcklund symmetries.

Definition 2. An operator

$$
X=\eta \frac{\partial}{\partial u}+\left(D_{x} \eta\right) \frac{\partial}{\partial u_{x}}+\left(D_{t} \eta\right) \frac{\partial}{\partial u_{t}}+\left(D_{x x} \eta\right) \frac{\partial}{\partial u_{x x}}+\cdots,
$$

where $\eta=\eta\left(t, x, u, u_{x}, u_{x x}, \ldots\right)$, will be called a nonclassical Lie-Bäcklund symmetry for a partial differential equation

$$
u_{t}=F\left(x, u, u_{x}, u_{x x}, \ldots\right),
$$

if

$$
\left.X\left(u_{t}-F\right)\right|_{\substack{u_{t}=F \\ \eta=0}}=0 .
$$


The equation (3) is the determining equation for nonclassical Lie-Bäcklund symmetries. This definition is well known and can be found in the paper by Zhdanov [7].

Theory of approximate point symmetries was developed by Baikov, Gazizov, Ibragimov in [1, 13]. They proposed to consider point symmetries in the form of formal power series

$$
X=\stackrel{0}{X}+\varepsilon \stackrel{1}{X}+\cdots+\varepsilon^{n} \stackrel{n}{X}+\cdots .
$$

Now we introduce approximate nonclassical Lie-Bäcklund symmetries.

Definition 3. An operator

$$
\begin{aligned}
X= & \left(\sum_{i=0}^{n} \varepsilon^{i} i^{i}\right) \frac{\partial}{\partial u}+\left(D_{x}\left(\sum_{i=0}^{n} \varepsilon^{i} \eta\right)\right) \frac{\partial}{\partial u_{x}}+\left(D_{t}\left(\sum_{i=0}^{n} \varepsilon^{i}{ }^{i}\right)\right) \frac{\partial}{\partial u_{t}} \\
& +\left(D_{x x}\left(\sum_{i=0}^{n} \varepsilon^{i} i^{i}\right)\right) \frac{\partial}{\partial u_{x x}}+\cdots,
\end{aligned}
$$

where $\stackrel{k}{\eta}=\stackrel{k}{\eta}\left(t, x, u, u_{x}, u_{x x}, \ldots\right), k=1,2, \ldots, n$ will be called an approximate nonclassical LieBäcklund symmetry (in the nth order order of precision) for an evolution partial differential equation with a small parameter:

$$
u_{t}=F\left(t, x, u, u_{x}, u_{x x}, \ldots\right)+\varepsilon G\left(t, x, u, u_{x}, u_{x x}, \ldots\right)+o(\varepsilon)
$$

if

$$
\left.X\left(u_{t}-F-\varepsilon G\right)\right|_{\sum_{i=0}^{n}=F+\varepsilon G}=o\left(\varepsilon^{n}\right) .
$$

The equation (5) is the determining equation for approximate nonclassical Lie-Bäcklund symmetries.

Recall that, by definition, the equality $\alpha(z, \varepsilon)=o\left(\varepsilon^{p}\right)$ is equivalent to the following condition:

$$
\lim _{\varepsilon \rightarrow 0} \frac{\alpha(z, \varepsilon)}{\varepsilon^{p}}=0 .
$$

Here $p$ is called the order of precision.

We will use the following theorem on stability of symmetries of the transport equation [1].

Theorem 1 (Baikov, Gazizov, Ibragimov). Any canonical Lie-Bäcklund symmetry

$$
\stackrel{0}{X}=\stackrel{0}{\eta} \frac{\partial}{\partial u}+\left(D_{x} \stackrel{0}{\eta}\right) \frac{\partial}{\partial u_{x}}+\left(D_{t} \stackrel{0}{\eta}\right) \frac{\partial}{\partial u_{t}}
$$

of the equation

$$
u_{t}=h(u) u_{x}
$$

gives rise to an approximate symmetry of the form (4) of the equation

$$
u_{t}=h(u) u_{x}+\varepsilon H\left(t, x, u, u_{x}, u_{x x}, \ldots\right)
$$

with an arbitrary order of precision in $\varepsilon$.

In other words, the equation (7) approximately inherits all the symmetries of the equation (6). 


\section{Approximate conditionally invariant solutions}

Now we introduce the definition of approximate conditionally invariant solutions:

Definition 4. An approximate solution of an equation

$$
u_{t}=F\left(t, x, u, u_{x}, u_{x x}, \ldots\right)+\varepsilon G\left(t, x, u, u_{x}, u_{x x}, \ldots\right)+o(\varepsilon)
$$

written in the form of a formal power series

$$
u=\sum_{i=0}^{\infty} \varepsilon^{i}{ }^{i}
$$

is called conditionally invariant under an approximate nonclassical symmetry $X$ (in the nth order order of precision), given by formula (4), if

$$
\sum_{j=0}^{\infty} \eta_{\eta}^{j}\left(\sum_{i=0}^{\infty} \varepsilon^{i} u\right)=o\left(\varepsilon^{n}\right) .
$$

As an example, we consider approximate nonclassical symmetries of the KdV equation

$$
u_{t}-u u_{x}-\varepsilon u_{x x x}=0 .
$$

Take the exact nonclassical Lie-Bäcklund symmetry of the transport equation:

$$
\stackrel{0}{X}=\stackrel{0}{\eta} \frac{\partial}{\partial u}+\cdots, \quad \stackrel{0}{\eta}=u_{x x} .
$$

It is easy to check that this is not a classical Lie-Bäcklund symmetry.

The corresponding approximate nonclassical Lie-Bäcklund symmetry of the approximate $\mathrm{KdV}$ equation (8) is written in the form

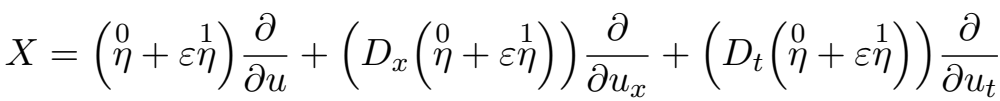

$$
\begin{aligned}
& +\left(D_{x x x}\left(\eta_{\eta}^{0}+\varepsilon \eta\right)\right) \frac{\partial}{\partial u_{x x x}} \text {. }
\end{aligned}
$$

From the determining equation (5) for $X$, it follows that

$$
\begin{aligned}
& \varepsilon^{0}: \stackrel{0}{\eta}=u_{x x}, \\
& \varepsilon^{1}: \frac{\partial}{\partial t} \stackrel{1}{\eta}-u_{x} \stackrel{1}{\eta}-u \frac{\partial}{\partial x} \stackrel{1}{\eta}+u_{x}^{2} \frac{\partial}{\partial u_{x}} \stackrel{1}{\eta}+3 u_{x} u_{x x} \frac{\partial}{\partial u_{x x}} \stackrel{1}{\eta} \\
& \quad+\left(3 u_{x x}^{2}+4 u_{x} u_{x x x}\right) \frac{\partial}{\partial u_{x x x}} \stackrel{1}{\eta}+\left(10 u_{x x} u_{x x x}+5 u_{x} u_{x x x x}\right) \frac{\partial}{\partial u_{x x x x}} \stackrel{1}{\eta} \\
& \quad+\left(10 u_{x x x}^{2}+15 u_{x x} u_{x x x x}+6 u_{x} u_{x x x x x}\right) \frac{\partial}{\partial u_{x x x x x}} \stackrel{1}{\eta}-u_{x x x x x}=0 .
\end{aligned}
$$

Whence we get

$$
\begin{gathered}
\stackrel{1}{\eta}=-F\left(u, x+u t, \frac{u_{x} t+1}{u_{x}},-\frac{u_{x x}}{u_{x}^{3}}, \frac{u_{x} u_{x x x}-3 u_{x} x^{2}}{u_{x}^{5}},-\frac{u_{x x x x} u_{x}^{2}-10 u_{x} u_{x x} u_{x x x}+15 u_{x x}^{3}}{u_{x}^{7}},\right. \\
\left.-\frac{105 u_{x x}^{4}-u_{x x x x x} u_{x}^{3}-105 u_{x x}^{2} u_{x} u_{x x x}+15 u_{x x} u_{x x x x} u_{x}^{2}+10 u_{x}^{2} u_{x x x}^{2}}{u_{x}^{9}}\right) u_{x}
\end{gathered}
$$




$$
+\frac{1}{4} \frac{u_{x x x x x}}{u_{x}}+\frac{-\frac{1}{2} u_{x x x}^{2}-\frac{3}{4} u_{x x} u_{x x x x}}{u_{x}^{2}}+\frac{7}{4} \frac{u_{x} x^{2} u_{x x x}}{u_{x}^{3}}-\frac{3}{4} \frac{u_{x x}^{4}}{u_{x}^{4}},
$$

where $F$ is an arbitrary function.

Note that the order of $\stackrel{1}{\eta}$ equals the sum of the orders of $\stackrel{0}{\eta}$ and the perturbation $G$ minus one. Here we consider an approximate conditionally invariant solution of the $\mathrm{KdV}$ equation (8) in the form:

$$
u=u^{0}+\varepsilon u^{1}+o(\varepsilon) .
$$

Conditional invariance under an approximate nonclassical symmetry (9) in the first order of precision is written as

$$
\stackrel{0}{\eta}\left(u^{0}+\varepsilon u^{1}\right)+\varepsilon \eta\left(u^{0}+\varepsilon u^{1}\right)=o(\varepsilon) .
$$

To compute an approximately invariant solution in the zero order of precision, we use the following reduction theorem [7].

Theorem 2. Suppose that an equation

$$
u_{t}=F\left(t, x, u, u_{x}, u_{x x}, \ldots, u_{N}\right), \quad u_{N}=\frac{\partial^{N} u}{\partial x^{N}}
$$

is conditionally invariant under a Lie-Bäcklund operator (2). Let

$$
u=f\left(t, x, C_{1}, C_{2}, \ldots, C_{N}\right)
$$

be a general solution of the equation $\eta\left(t, x, u, u_{1}, \ldots, u_{N}\right)=0$. Then the Ansatz

$$
u=f\left(t, x, \varphi_{1}(t), \varphi_{2}(t), \ldots, \varphi_{N}(t)\right),
$$

where $\varphi_{j}(t), j=1,2, \ldots, N$, are arbitrary smooth functions, reduces the partial differential equation $u_{t}=F$ to a system of $N$ ordinary differential equations for the functions $\varphi_{j}(t), j=$ $1,2, \ldots, N$.

There is a nice consequence of this theorem.

Corollary 1. Suppose an equation

$$
u_{t}=F\left(t, x, u, u_{x}, u_{x x}, \ldots\right)+\varepsilon G\left(t, x, u, u_{x}, u_{x x}, \ldots\right)+o(\varepsilon)
$$

admits an approximate Lie-Bäcklund operator $X$, given by the formula (4) with $n=1$. Let

$$
\stackrel{0}{u}=f\left(t, x, C_{1}, C_{2}, \ldots, C_{N}\right), \quad \stackrel{1}{u}=g\left(t, x, C_{1}, \ldots, C_{N+M}\right)
$$

be a general solution of the equation

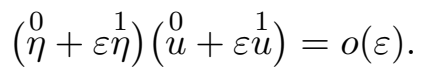

Then the Ansatz

$$
\begin{aligned}
u= & f\left(t, x, \varphi_{1}(t), \varphi_{2}(t), \ldots, \varphi_{N}(t)\right) \\
& +\varepsilon g\left(t, x, \varphi_{1}(t), \varphi_{2}(t), \ldots, \varphi_{N}(t), \psi_{1}(t), \psi_{2}(t), \ldots, \psi_{M}(t)\right),
\end{aligned}
$$

reduces the equation $u_{t}=F+\varepsilon G$ into a system of $N+M$ ordinary differential equations for $\varphi_{j}(t)$, $j=1,2, \ldots, N$, and $\psi_{k}(t), k=1,2, \ldots, M$. 
Example 1. Take a nonclassical symmetry $(2)(\eta=\stackrel{0}{\eta})$ of the transport equation

$$
u_{t}=u u_{x}
$$

where

$$
\stackrel{0}{\eta}=u_{x x}
$$

Applying the operator (2) to the equation (12) in the zero order of precision, we have $\stackrel{0}{\eta}\left(u^{0}\right)=0$, whence we get $u^{0}=A x+B$.

By Reduction Theorem, we substitute

$$
u^{0}=A(t) x+B(t)
$$

to the transport equation (12) and get

$$
\dot{A}=A^{2}, \quad \dot{B}=A B .
$$

A general solution has the form:

$$
A=-\frac{1}{t+a}, \quad B=\frac{b}{t+a},
$$

where $a, b$ are constants.

Thus we get

$$
u^{0}=\frac{b-x}{t+a} .
$$

Take $\stackrel{1}{\eta}$ as in (10) with

$$
F(u)=p e^{u} .
$$

where $p$ is a constant. From (11) it follows that

$$
u_{x x}^{1}-\frac{p}{t+a} e^{\frac{b-x}{t+a}}=0
$$

and

$$
u^{1}=p(t+a) e^{\frac{b-x}{t+a}}+C x+D .
$$

Take the approximate solution

$$
u=\frac{b-x}{t+a}+\varepsilon\left(p(t)(t+a) e^{\frac{b-x}{t+a}}+C(t) x+D(t)\right)
$$

and substitute it into the $\mathrm{KdV}$ equation (8). We get three first order ODE for $C(t), D(t), p(t)$ :

$$
\dot{C}=-\frac{2 C}{t+a}, \quad \dot{D}=\frac{b C-D}{t+a}, \quad \dot{p}=\frac{-2 p}{t+a} .
$$

A general solution of the system can be written as

$$
C(t)=\frac{c_{3}}{b(t+a)^{2}}, \quad D(t)=\frac{c_{2} t+c_{2} a+c_{3}}{(t+a)^{2}}, \quad p(t)=\frac{c_{1}}{(t+a)^{2}}
$$

where $c_{1}, c_{2}, c_{3}$ are constants.

Finally, we get the following solution of the $\mathrm{KdV}$ equation in the first order of precision:

$$
u=\frac{b-x}{t+a}+\varepsilon\left(\frac{c_{1}}{t+a} e^{\frac{b-x}{t+a}}+\frac{c_{3} x}{b(t+a)^{2}}+\frac{c_{2} t+c_{2} a+c_{3}}{(t+a)^{2}}\right) .
$$


We use the following proposition to construct nonclassical symmetries.

Proposition 1. Let

$$
X=\eta \frac{\partial}{\partial u}+\cdots, \quad \eta=\eta\left(t, x, u, u_{x}, u_{x x}, \ldots\right),
$$

be a classical Lie-Bäcklund symmetry for a first order PDE

$$
F\left(t, x, u, u_{x}, u_{t}\right)=0 .
$$

For any function $f=f\left(t, x, u, u_{x}, u_{x x}, \ldots\right)$, the operator

$$
\stackrel{*}{X}=\stackrel{*}{\eta} \frac{\partial}{\partial u}+\cdots, \quad \stackrel{*}{\eta}=f \eta,
$$

is a nonclassical Lie-Bäcklund symmetry for (13).

Example 2. Now we consider an example of finding symmetries of the KdV equation with a small parameter (8) and construct its approximate solution. We have a classical Lie-Bäcklund symmetry

$$
X=\stackrel{0}{\eta} \frac{\partial}{\partial u}+D_{x}(\stackrel{0}{\eta}) \frac{\partial}{\partial u_{x}}+D_{t}(\stackrel{0}{\eta}) \frac{\partial}{\partial u_{t}}
$$

of the transport equation (12), where

$$
\stackrel{0}{\eta}=u_{x} \Phi\left(u, x+u t, \frac{u_{x} t+1}{u_{x}},-\frac{u_{x x}}{u_{x}^{3}}, \frac{u_{x} u_{x x x}-3 u_{x x}^{2}}{u_{x}^{5}}\right) .
$$

By Proposition $1, \stackrel{0}{\eta}=u_{x} u_{x x x}-3 u_{x x}^{2}$ is a nonclassical Lie-Bäcklund symmetry of the transport equation (12). Now we take operator (9). Applying the operator $X$ to the equation (8), we get the following equations in the zero and first orders of precision in $\varepsilon$ :

$$
\begin{aligned}
& \varepsilon^{0}: \stackrel{0}{\eta}=u_{x} u_{x x x}-3 u_{x x}^{2} \\
& \varepsilon^{1}: \frac{\partial}{\partial t} \stackrel{1}{\eta}-u_{x} \stackrel{1}{\eta}-u \frac{\partial}{\partial x} \stackrel{1}{\eta}+u_{x}^{2} \frac{\partial}{\partial u_{x}} \stackrel{1}{\eta}+3 u_{x} u_{x x} \frac{\partial}{\partial u_{x x}} \stackrel{1}{\eta}+\left(3 u_{x x}^{2}+4 u_{x} u_{x x x}\right) \frac{\partial}{\partial u_{x x x}} \stackrel{1}{\eta} \\
& +\left(10 u_{x x} u_{x x x}+5 u_{x} u_{x x x x}\right) \frac{\partial}{\partial u_{x x x x}} \stackrel{1}{\eta}+\left(10 u_{x x x}^{2}+15 u_{x x} u_{x x x x}+6 u_{x} u_{x x x x x}\right) \frac{\partial}{\partial u_{x x x x x}} \stackrel{1}{\eta} \\
& +\left(35 u_{x x x} u_{x x x x}+21 u_{x x} u_{x x x x x}+7 u_{x} u_{x x x}\right) \frac{\partial}{\partial u_{x x x x x x}} \stackrel{1}{\eta}+14 u_{x x x} u_{x x x x} \\
& +3 u_{x x} u_{x x x x x}-u_{x} u_{x x x x x x}=0 .
\end{aligned}
$$

From the last equation, we find

$$
\begin{aligned}
\stackrel{1}{\eta}= & -F\left(u, x+u t, \frac{u_{x} t+1}{u_{x}},-\frac{u_{x x}}{u_{x}^{3}}, \frac{u_{x} u_{x x x}-3 u_{x x}^{2}}{u_{x}^{5}},-\frac{15 u_{x x}^{3}+u_{x x x x} u_{x}^{2}-10 u_{x x} u_{x} u_{x x x}}{u_{x}^{7}},\right. \\
& -\frac{105 u_{x x}^{4}-u_{x x x x x} u_{x}^{3}-105 u_{x x}^{2} u_{x} u_{x x x}+15 u_{x x} u_{x x x x} u_{x}^{2}+10 u_{x}^{2} u_{x x x}^{2}}{u_{x x x}^{9}}, \\
& -\frac{945 u_{x x}^{5}-1260 u_{x x}^{3} u_{x} u_{x x x}+280 u_{x x} u_{x}^{2} u_{x x x}^{2}+210 u_{x x}^{2} u_{x x x x} u_{x}^{2}-21 u_{x x} u_{x x x x x} u_{x}^{3}}{u_{x}^{11}} \\
& \left.+\frac{-35 u_{x}^{3} u_{x x x} u_{x x x x}+u_{x x x x x x} u_{x}^{4}}{u_{x}^{11}}\right) u_{x}+\frac{1}{6} u_{x x x x x x}+\left(-\frac{13}{14} u_{x x} u_{x x x x x}-\frac{17}{6} u_{x x x} u_{x x x x}\right) u_{x}^{-1}
\end{aligned}
$$




$$
+\left(\frac{395}{84} u_{x x} u_{x x x}^{2}+\frac{157}{56} u_{x x}^{2} u_{x x x x}\right) u_{x}^{-2}-\frac{25}{4} \frac{u_{x x}^{3} u_{x x x}}{u_{x}^{3}}+\frac{15}{8} \frac{u_{x x}^{5}}{u_{x}^{4}},
$$

where $F$ is an arbitrary function. The invariance condition of a solution

$$
u=\stackrel{0}{u}+\varepsilon \stackrel{1}{u}+\cdots
$$

in the first order of precision is written as

$$
(\stackrel{0}{\eta}+\varepsilon \stackrel{1}{\eta})(\stackrel{0}{u}+\varepsilon \stackrel{1}{u})=o(\varepsilon)
$$

If we substitute $\stackrel{0}{\eta}, \stackrel{1}{\eta}$ to the equation (14), we obtain in the zero and first orders of precision by $\varepsilon$ equations for $\stackrel{0}{u}$ and $\stackrel{1}{u}$ :

$$
\begin{aligned}
& \varepsilon^{0}: \stackrel{0}{\eta}(\stackrel{0}{u})=0 \\
& \varepsilon^{1}: \stackrel{1}{u_{x}} \stackrel{0}{u}_{x x x}+\stackrel{0}{u_{x}} \stackrel{1}{u_{x x x}}-6 u_{x x} \stackrel{0}{u}_{x x}+\stackrel{1}{\eta}(\stackrel{0}{u})=0 .
\end{aligned}
$$

We find

$$
\stackrel{0}{u}=2 \sqrt{t^{2}+t-x}-2 t-1
$$

and substitute it in the second equation:

$$
-\frac{\stackrel{1}{u}_{x x x}}{\sqrt{t^{2}+t-x}}-\frac{3 u_{x}}{4\left(t^{2}+t-x\right)^{5 / 2}}+3 \frac{\stackrel{1}{u}_{x x}}{\left(t^{2}+t-x\right)^{3 / 2}}+c\left(t^{2}+t-x\right)^{-11 / 2}=0,
$$

where $c$ is a constant, depending on the choice of $F$. The equation (15) is an ordinary differential equation and has the following solution:

$$
\stackrel{1}{u}=\frac{2 c}{15\left(t^{2}+t-x\right)^{2}}+F_{1}(t)+\frac{F_{2}(t)}{\sqrt{t^{2}+t-x}}+F_{3}(t) \sqrt{t^{2}+t-x} .
$$

If we substitute $u=\stackrel{0}{u}+\varepsilon \stackrel{1}{u}$ in (8) we obtain a system of ordinary differential equations for finding $F_{1}(t), F_{2}(t), F_{3}(t)$ :

$$
\dot{F}_{1}=-2 F_{3}, \quad \dot{F}_{2}=-F_{1}, \quad \dot{F}_{3}=0,
$$

which has the solution:

$$
F_{1}=-2 A t+B, \quad F_{2}=A t^{2}-B t+C, \quad F_{3}=A,
$$

where $A, B, C$ are arbitrary constants, $c=\frac{1}{4}$. Finally, we find the solution of (8):

$$
\begin{aligned}
u= & 2 \sqrt{t^{2}+t-x}-2 t-1 \\
& +\varepsilon\left(\frac{1}{4}\left(t^{2}+t-x\right)^{2}+(-2 A t+B)+\frac{A t^{2}-B t+C}{\sqrt{t^{2}+t-x}}+A \sqrt{t^{2}+t-x}\right),
\end{aligned}
$$

where $A, B, C$ are arbitrary constants.

Example 3. Now we consider an example of finding of symmetries of the nonintegrable equation

$$
u_{t}=u u_{x}+u_{x x x}^{2}
$$


and construct its approximate solution. Using the criteria of integrability, it can be checked that the equation (16) is nonintegrable [11].

As in Example 2, take a nonclassical Lie-Bäcklund symmetry of the transport equation (12) with $\stackrel{0}{\eta}=u_{x} u_{x x x}-3 u_{x x}^{2}$. Applying the operator, given by (9), to the equation (16) we get in the zero and first orders of precision by $\varepsilon$ :

$$
\begin{aligned}
\varepsilon^{0}: & \stackrel{0}{\eta}=u_{x} u_{x x x}-3 u_{x x}^{2} \\
\varepsilon^{1}: & \frac{\partial}{\partial t} \stackrel{1}{\eta}-u_{x} \stackrel{1}{\eta}-u \frac{\partial}{\partial x} \stackrel{1}{\eta}+u_{x}^{2} \frac{\partial}{\partial u_{x}} \stackrel{1}{\eta}+3 u_{x} u_{x x} \frac{\partial}{\partial u_{x x}} \stackrel{1}{\eta}+\left(3 u_{x x}^{2}+4 u_{x} u_{x x x}\right) \frac{\partial}{\partial u_{x x x}} \stackrel{1}{\eta} \\
& +\left(10 u_{x x} u_{x x x}+5 u_{x} u_{x x x x}\right) \frac{\partial}{\partial u_{x x x x}} \stackrel{1}{\eta}+\left(10 u_{x x x}^{2}+15 u_{x x} u_{x x x x}+6 u_{x} u_{x x x x x}\right) \frac{\partial}{\partial u_{x x x x x}} \stackrel{1}{\eta} \\
& +\left(35 u_{x x x} u_{x x x x}+21 u_{x x} u_{x x x x x}+7 u_{x} u_{x x x}\right) \frac{\partial}{\partial u_{x x x x x x}} \stackrel{1}{\eta} \\
& +2 u_{x x x}\left(14 u_{x x x} u_{x x x x}+3 u_{x x} u_{x x x x x}-u_{x} u_{x x x x x x}\right)=0 .
\end{aligned}
$$

From the last equation, we find

$$
\begin{aligned}
\eta= & -F\left(u, x+u t, \frac{u_{x} t+1}{u_{x}},-\frac{u_{x x}}{u_{x}^{3}}, \frac{u_{x} u_{x x x}-3 u_{x x}^{2}}{u_{x}^{5}},-\frac{15 u_{x x}^{3}+u_{x x x x} u_{x}^{2}-10 u_{x x} u_{x} u_{x x x}}{u_{x}^{7}},\right. \\
& -\frac{105 u_{x x}^{4}-u_{x x x x x} u_{x}^{3}-105 u_{x x}^{2} u_{x} u_{x x x}+15 u_{x x} u_{x x x x} u_{x}^{2}+10 u_{x}^{2} u_{x x x}^{2}}{u_{x x x}^{9}}, \\
& -\frac{945 u_{x x}^{5}-1260 u_{x x}^{3} u_{x} u_{x x x}+280 u_{x x} u_{x}^{2} u_{x x x}^{2}+210 u_{x x}^{2} u_{x x x x} u_{x}^{2}-21 u_{x x} u_{x x x x x} u_{x}^{3}}{u_{x}^{11}} \\
& \left.+\frac{-35 u_{x}^{3} u_{x x x} u_{x x x x}+u_{x x x x x x} u_{x}^{4}}{u_{x}^{11}}\right) u_{x}+\frac{1}{5} u_{x x x} u_{x x x x x x} \\
& -\left(\frac{51}{55} u_{x x x} u_{x x} u_{x x x x x}-\frac{3}{55} u_{x x}^{2} u_{x x x x x x}-\frac{35}{11} u_{x x x}^{2} u_{x x x x}\right) u_{x}^{-1} \\
& +\left(\frac{32}{11} u_{x x}^{2} u_{x x x} u_{x x x x}+\frac{113}{33} u_{x x} u_{x x x}^{3}+\frac{18}{55} u_{x x}^{3} u_{x x x x x}\right) u_{x}^{-2} \\
& +\left(-\frac{695}{143} u_{x x}^{3} u_{x x x}^{2}-\frac{150}{143} u_{x x}^{4} u_{x x x x}\right) u_{x}^{-3}+\frac{405}{143} u_{x x}^{5} u_{x x x} u_{x}^{-4}-\frac{81}{143} u_{x x}^{7} u_{x}^{-5} .
\end{aligned}
$$

where $F$ is an arbitrary function. Now we find an approximate solution of the equation (8) in the form $u=\stackrel{0}{u}+\varepsilon \stackrel{1}{u}+o(\varepsilon)$. The invariance condition in the first order of precision is written as:

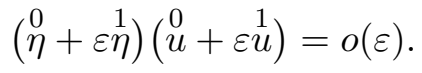

If we substitute $\stackrel{0}{\eta}, \stackrel{1}{\eta}$ to the equation (17), we obtain in the zero and first orders by $\varepsilon$ equations for $\stackrel{0}{u}$ and $\stackrel{1}{u}$ :

$$
\begin{aligned}
& \varepsilon^{0}: \stackrel{0}{\eta}(\stackrel{0}{u})=0 \\
& \varepsilon^{1}: \stackrel{1}{u}_{x}{\stackrel{0}{u_{x x x}}}+\stackrel{0}{u_{x}} \stackrel{1}{u_{x x x}}-6{ }_{u_{x x}} \stackrel{0}{u}_{x x}+\stackrel{1}{\eta}(\stackrel{0}{u})=0 .
\end{aligned}
$$

From the first equation, we get

$$
\stackrel{0}{u}=2 \sqrt{t^{2}+t-x}-2 t-1,
$$


and, substituting this expression into the second equation, we obtain

$$
-\frac{\stackrel{1}{u}_{x x x}}{\sqrt{t^{2}+t-x}}-\frac{3 u_{x}}{4\left(t^{2}+t-x\right)^{5 / 2}}+3 \frac{\stackrel{1}{u}_{x x}}{\left(t^{2}+t-x\right)^{3 / 2}}+c\left(t^{2}+t-x\right)^{-8}=0
$$

where $c$ is a constant depending on the choice of $F$. The equation (18) is an ordinary differential equation and has the following solution:

$$
\stackrel{1}{u}=\frac{c}{90}\left(t^{2}+t-x\right)^{-9 / 2}+(-2 A t+B)+\frac{A t^{2}-B t+C}{\sqrt{t^{2}+t-x}}+A \sqrt{t^{2}+t-x} .
$$

If we substitute $u=\stackrel{0}{u}+\varepsilon \dot{1}$ in the equation (16) we obtain the system of ordinary differential equations for finding $F_{1}(t), F_{2}(t), F_{3}(t)$ :

$$
\dot{F}_{1}=-2 F_{3}, \quad \dot{F}_{2}=-F_{1}, \quad \dot{F}_{3}=0,
$$

which has the solution:

$$
F_{1}=-2 A t+B, \quad F_{2}=A t^{2}-B t+C, \quad F_{3}=A .
$$

Therefore, the solution $u$ has the form:

$$
\begin{aligned}
u= & 2 \sqrt{t^{2}+t-x}-2 t-1 \\
& +\varepsilon\left(\frac{405}{64}\left(t^{2}+t-x\right)^{-9 / 2}+(-2 A t+B)+\frac{A t^{2}-B t+C}{\sqrt{t^{2}+t-x}}+A \sqrt{t^{2}+t-x}\right),
\end{aligned}
$$

where $A, B, C$ are arbitrary constants.

Remark 1. One can show that the approximate symmetries constructed in the above examples remain stable in any higher order of precision. However, we do not know whether any nonclassical symmetry of an evolution partial differential equation with a small parameter is stable in any order of precision.

\section{Conclusion}

The methods developed in this paper can be applied to larger classes of partial differential equations with a small parameter, not only to the evolution ones. For instance, in the paper [12] it is shown that classical approximate Lie-Bäcklund symmetries of the Boussinesq equation with a small parameter can be constructed, starting from the exact Lie-Bäcklund symmetries of the linear wave equation. It is quite possible that these results can be extended to non-classical approximate symmetries of the Boussinesq equation.

From the other side, one should note that stability property of approximate classical symmetries holds only for a very restricted class of partial differential equations with a small parameter, mainly, for those, which have very nice symmetry properties in the zero order of precision. The class of non-classical symmetries is much larger than the class of classical symmetries. Therefore, one can hardly expect to have some general theorems on stability of non-classical symmetries. This means that we will have to investigate separately stability properties of non-classical symmetries in each particular case.

All the computations have been made with the help of Maple. 
[1] Baikov V.A., Gazizov R.K., Ibragimov N.Kh., Perturbation methods in group analysis, Current Problems in Mathematics. Newest Results, Vol. 34, Moscow, VINITI, 1989, 85-147 (English transl.: J. Soviet Math., 1991, V.55, 1450-1490).

[2] Bluman G.W., Cole J.D., The general similarity solutions of the heat equation, J. Math. Mech., 1969, V.18, 1025-1042.

[3] Olver P.J., Rosenau P., The construction of special solutions to partial differential equation, Phys. Lett. A, 1986, V.114, 107-112.

[4] Clarkson P.A., Kruskal M., New similarity reductions of the Boussinesq equation, J. Math. Phys., 1989, V.30, 2201-2213.

[5] Fushchych W.I., Shtelen W.M., Serov N.I., Symmetry analysis and exact solutions of nonlinear equations of mathematical physics, Kyiv, Naukova Dumka, 1989 (English transl.: Dordrecht, Kluwer, 1993).

[6] Fokas A.S., Liu Q.M., Generalized conditional symmetries and exact solutions of non-integrable equations, Teor. Mat. Fiz., 1994, V.99, 263-277 (English transl.: Theor. Math. Phys., 1994, V.99, 571-582).

[7] Zhdanov R.Z., Conditional Lie-Bäcklund symmetry and reduction of evolution equations, J. Phys. A: Math. Gen., 1995, V.28, 3841-3850.

[8] Mahomed F.M., Qu C., Approximate conditional symmetries for partial differential equations, J. Phys. A: Math. Gen., 2000, V.33, 343-356.

[9] Kara A.F., Mahomed F.M., Qu C., Approximate potential symmetries for partial differential equations, J. Phys. A: Math. Gen., 2000, V.33, 6601-6613.

[10] Olver P.J., Vorob'ev E.M., Nonclassical and conditional symmetries, in CRC Handbook of Lie Group Analysis of Differential Equations, Vol. 3, Editor N.H. Ibragimov, Boca Raton, Florida, CRC Press, 1996, 291-328.

[11] Sokolov V.V., Shabat A.B., Classification of integrable evolution equations, in Mathematical Physics Reviews, Vol. 4, Soviet Sci. Rev. Sect. C Math. Phys. Rev., 4, Chur, Harwood Academic Publ., 1984, 221-280.

[12] Baikov V.A., Kordyukova S.A., Approximate symmetries of the Boussinesq equation, Quaest. Math., 2003, V.26, 1-14.

[13] Baikov V.A., Gazizov R.K., Ibragimov N.Kh., Approximate transformation groups and deformations of symmetry Lie algebras, Chapter 2, in CRC Handbook of Lie Group Analysis of Differential Equation, Vol. 3, Editor N.H. Ibragimov, Boca Raton, Florida, CRC Press, 1996. 